\title{
Template-Free Synthesis and Enhanced Photocatalytic Performance of Uniform BiOCI Flower-Like Microspheres
}

\author{
Fei Chang ${ }^{1, *}$, Yunchao Xie ${ }^{1}$, Juan Chen ${ }^{1}$, Jieru Luo ${ }^{1}$, Chenlu Li ${ }^{1}$, \\ Xuefeng $\mathrm{Hu}^{2, *}$, and Bin $\mathrm{Xu}^{3}$ \\ ${ }^{1}$ School of Environment and Architecture, University of Shanghai for Science and Technology, \\ Shanghai 200093, P. R. China \\ ${ }^{2}$ Key Laboratory of Coastal Zone Environmental Processes and Ecological Remediation, Yantai Institute of \\ Coastal Zone Research, Chinese Academy of Sciences, Yantai, Shandong 264003, P. R. China \\ ${ }^{3}$ Inspection and Quarantine Technology Center, Hainan Entry-Exit Inspection and Quarantine Bureau, \\ Haikou, Hainan 570311, P. R. China
}

\begin{abstract}
Preparation of uniform $\mathrm{BiOCl}$ flower-like microspheres was facilely accomplished through a simple protocol involving regulation of $\mathrm{pH}$ value in aqueous with sodium hydroxide in the presence of $n$-propanol. The as-prepared samples were characterized by a collection of techniques, such as X-ray diffraction (XRD), scanning electron microscopy (SEM), transmission electron microscopy (TEM), energy dispersive X-ray spectroscopy (EDX), UV-vis diffuse reflectance spectroscopy (UV-vis DRS), and nitrogen adsorption-desorption isotherms. Based upon the SEM analyses, uniform microspheres could be formed with coexistence of some fragments of $\mathrm{BiOCl}$ nanosheets without $n$-propanol. The addition of appropriate amount of $n$-propanol was beneficial to provide $\mathrm{BiOCl}$ samples containing only flower-like microspheres, which were further subjected to the photocatalytic measurements towards Rhodamine $B$ in aqueous under visible light irradiation and exhibited the best catalytic performance among all samples tested. In addition, the photocatalytic process was confirmed to undergo through a photosensitization pathway, in which superoxide radicals $\left({ }^{\circ} \mathrm{O}_{2}^{-}\right)$ played critical roles.
\end{abstract}

Keywords: $\mathrm{BiOCl}$, Microspheres, $n$-Propanol, Photocatalytic, Rhodamine B.

\section{INTRODUCTION}

Semiconductor-based photocatalysis is a promising and suitable technique towards environmental remediation since it is capable of complete remove of organic pollutants under mild conditions. ${ }^{1-3}$ Among numerous photocatalyts researched, bismuth oxychloride $(\mathrm{BiOCl})$, belonging to the V-VI-VII main group, has recently attracted growing attention. ${ }^{4-6}$ It crystallizes in a tetragonal matlockite structure consisting of $\left[\mathrm{Bi}_{2} \mathrm{O}_{2}\right]^{2+}$ slabs interleaved with double chloride anions layers, which is favorable for an efficient separation of photoinduced electron-hole pairs. ${ }^{7,8}$ In addition, $\mathrm{BiOCl}$ is an indirectband-gap semiconductor, featuring slow recombination rate of electron-hole pairs. With both merits in structure,

\footnotetext{
*Authors to whom correspondence should be addressed.
}

$\mathrm{BiOCl}$ is regarded as an effective catalyst that is in some cases more powerful than anatase $\mathrm{TiO}_{2}$ over degrading organic pollutants. ${ }^{4}$

Hierarchical nanostructured $\mathrm{BiOCl}$ with controllable sizes and morphologies is desired for the purpose of enhancing photocatalytic performance. ${ }^{6,8}$ Hereby, two dimensional (2D) $)^{9-11}$ and three dimensional (3D) ${ }^{12-14}$ $\mathrm{BiOCl}$ nanostructures have been prepared through a variety of synthetic protocols. 3D hierarchical nanostructures have the advantages of both microstructure and nanostructure, such as the high crystallinity, high surface-tovolume ratio, strong light-harvesting ability, and abundant electronic transport paths, ensuring the improvement of photocatalytic capability. ${ }^{15,16}$ Zhang et al. synthesized $\mathrm{BiOCl}$ microflowers and hollow microspheres exhibiting excellent photocatalytic degradation capability over dye 
Rhodamine B (RhB). ${ }^{12}$ Lei et al. reported that 3D hierarchical structured $\mathrm{BiOCl}$ showed higher photocatalytic efficiency towards methyl orange than $2 \mathrm{D}$ nanoplates. ${ }^{13} \mathrm{Zhu}$ et al. demonstrated that $3 \mathrm{D} \mathrm{BiOCl}$ hierarchitectures were beneficial for photocatalysis in comparison with $\mathrm{BiOCl}$ nanofibers. ${ }^{17}$ Although well-defined hierarchical $\mathrm{BiOCl}$ can be readily fabricated, high reaction temperature and toxic/expensive organics are generally unavoidable during the hydrothermal or solvothermal syntheses. As a result, facile synthetic protocols without toxic/expensive organics under mild conditions are highly required to construct $\mathrm{BiOCl}$ hierarchitectures.

In our previous work, uniform $\mathrm{BiOCl}$ flower-like microspheres were synthesized through a polyvinyl alcoholassisted hydrothermal process and showed satisfactory photocatalytic performance. ${ }^{18}$ To continue the research, in this investigation we reported the facile construction of uniform BiOCl flower-like microspheres through simple regulation of $\mathrm{pH}$ value with sodium hydroxide in the presence of $n$-propanol at ambient temperature. The $\mathrm{BiOCl}$ samples with uniform flower-like structures exhibited enhanced photocatalytic performance against $\mathrm{RhB}$ in aqueous through a photosensitization pathway, in which superoxide radicals $\left({ }^{\circ} \mathrm{O}_{2}^{-}\right)$played critical roles.

\section{EXPERIMENTAL DETAILS}

\subsection{Preparation of $\mathrm{BiOCl}$ Samples}

All chemical reagents were analytically pure and used directly for experiments without any purification. Uniform $\mathrm{BiOCl}$ flower-like microspheres were prepared as follows. In a typical synthesis, $\mathrm{Bi}\left(\mathrm{NO}_{3}\right)_{3} \cdot 5 \mathrm{H}_{2} \mathrm{O}(3.0 \mathrm{~g})$ was dissolved in a $\mathrm{HCl}$ aqueous $(2 \mathrm{M}, 25 \mathrm{~mL})$ under vigorous stir to provide a transparent solution. A mixture of distilled water $(20 \mathrm{~mL})$ and $n$-propanol $(10 \mathrm{~mL})$ was dropped into the above solution, followed by careful addition of $\mathrm{NaOH}$ aqueous (4 M, $20 \mathrm{~mL}$ ). The resultant white suspension was allowed to stir at $20{ }^{\circ} \mathrm{C}$ for $0.5 \mathrm{~h}$ and then age at the same temperature for $6 \mathrm{~h}$. After reaction, the precipitate was collected, washed with deionized water and ethanol for several times, and dried at $80{ }^{\circ} \mathrm{C}$ overnight to give a target sample, denoted as BOC10. Other samples were prepared under a similar protocol except the variation of $n$-propanol volumes and denoted as BOC $x$, where $x$ referred to the volumes of $n$-propanol involved. $N$-doped $\mathrm{TiO}_{2}{ }^{19}$ and reference $\mathrm{BiOCl}^{18}$ were also prepared and subjected to photocatalytic measurements for comparison.

\subsection{Characterization}

The powder X-ray diffraction (XRD) patterns were recorded on a Bruker D8 Advance X-ray diffractometer using a $\mathrm{Cu} \mathrm{K} \alpha$ radiation source $(\lambda=1.05406 \AA)$. The general morphology of samples was observed by Hitachi S-4800 FEG scanning electron microscope (SEM) under a working voltage of $200 \mathrm{kV}$. Transmission electron microscopy and energy-dispersive X-ray spectroscopy were obtained on a JEOL JEM-2011 transmission electron microscope (TEM) equipped with Oxford Instruments INCA energy-dispersive X-ray spectroscope (EDX). The optical property of prepared samples was measured through UV-vis diffuse reflectance spectroscopy (UV-vis DRS, Hitachi U-4100) by using $\mathrm{BaSO}_{4}$ as a reference. Nitrogen adsorption-desorption isotherms at $77 \mathrm{~K}$ were performed on an ASAP 2010 volumetric adsorption analyzer.

\subsection{Photocatalytic Performance Evaluation}

$\mathrm{RhB}$ was selected as a probe pollutant to evaluate photocatalytic performance of as-synthesized samples in a LIMX-VII apparatus by Bylabo Precision Instrument Co. Ltd. (Xi'an, China). The visible light was supplied from a $400 \mathrm{~W}$ halogen lamp (Institute for Electric Light Sources, China) through a $420 \mathrm{~nm}$ cutoff glass filter. The suspension containing $\mathrm{RhB}$ aqueous $(40 \mathrm{mg} / \mathrm{L}, 40 \mathrm{~mL})$ and catalyst $(10 \mathrm{mg}$ ) was magnetically stirred for $1 \mathrm{~h}$ in dark to ensure an adsorption-desorption equilibrium. During irradiation, $2 \mathrm{~mL}$ aliquot was sampled at certain time intervals, diluted, and centrifuged to remove any suspended catalyst particles prior to analysis at $554 \mathrm{~nm}$ using a T6 UV-vis spectrophotometer (Beijing Purkinje General Instrument Co., Ltd., China). The total organic carbon concentration was measured by a TOC analyzer (AnalytikJena multi N/C 3100 TOC, Germany). 1.0 mM isopropanol (IPA) ${ }^{20}$ and benzoquinone $(\mathrm{BQ})^{5}$ were added to the photocatalytic system to capture hydroxyl radicals $\left({ }^{\circ} \mathrm{OH}\right)$ and superoxide radicals $\left({ }^{\circ} \mathrm{O}_{2}^{-}\right)$, respectively.

\section{RESULTS AND DISCUSSION}

\subsection{Crystalline}

The crystallinity and phase purity of $\mathrm{BiOCl}$ samples prepared with different $n$-propanol volumes were examined by XRD patterns. As shown in Figure 1, all diffraction peaks

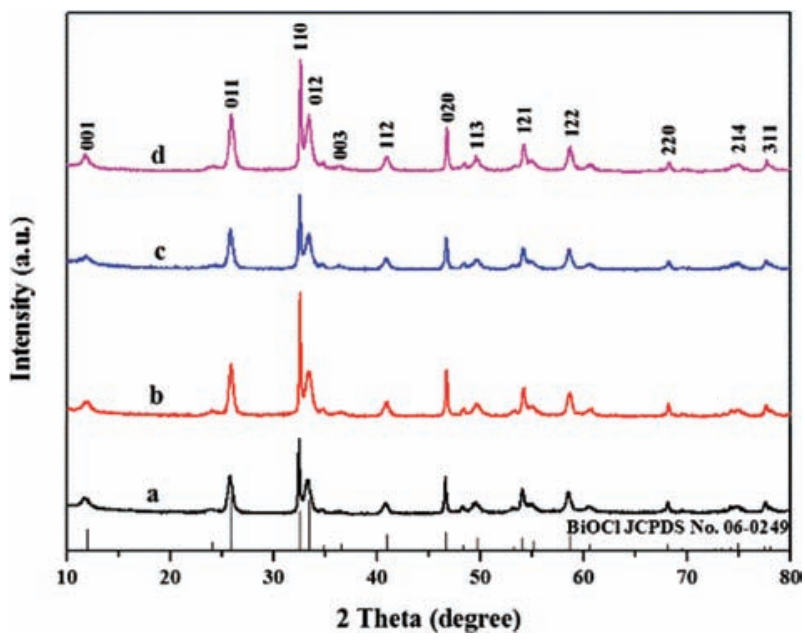

Figure 1. XRD patterns of the as-synthesized samples (a) BOC0; (b) BOC5; (c) BOC10; (d) BOC20. 
can be unambiguously indexed to the tetragonal phase of $\mathrm{BiOCl}$ on the basis of the standard card JCPDS 06-0249. ${ }^{21}$ No diffraction peaks of other impurities are observable, revealing the high purity of as-synthesized samples. The narrow and sharp peaks indicate that all as-prepared $\mathrm{BiOCl}$ samples are well crystallized. Further observations find that the intensity ratio of the (110) peak to the (012) peak of as-synthesized samples around 1.5 (BOC20 around 0.9) is obviously larger than the data 0.8 of the JCPDS card, suggesting that the crystal structure of $\mathrm{BiOCl}$ had special anisotropic growth along the (110) plane. $^{6}$

\subsection{Morphology and Microstructure}

The sizes and micromorphologies of microspheres BOC10 and other BiOCl samples were characterized by SEM and TEM, as displayed in Figure 2.

From the SEM image in Figure 2(C), the sample BOC10 contains regular and uniform flower-like microspheres in morphology. These flower-like microspheres with an average diameter of nearly $1.5 \mu \mathrm{m}$ are entirely assembled by numerous $\mathrm{BiOCl}$ nanosheets, clearly shown in Figures 2(C) and (E). In the absence or presence of unsuitable amount of $n$-propanol, flower-like microspheres can even be produced with coexistence of some fragments of $\mathrm{BiOCl}$ nanosheets, as seen the circles labeled in Figures 2(A), (B), and (D). It is obvious that the addition of appropriate amount of $n$-propanol favored to promote the formation of flower-like microspheres through combination and assembly of $\mathrm{BiOCl}$ nanosheets. From the images, we regarded that $10 \mathrm{~mL} n$-propanol were suitable to produce uniform microspheres. Less than $10 \mathrm{~mL}$, such as 0 or $5 \mathrm{~mL}$, might be insufficient to exert the role and beyond $10 \mathrm{~mL}$, like $20 \mathrm{~mL}$, might obstruct other than combine $\mathrm{BiOCl}$ nanosheets. The surface chemical composition of sample BOC10 was also tested by EDX. It reveals that the uniform $\mathrm{BiOCl}$ flower-like microspheres consist of only three elements $\mathrm{Bi}, \mathrm{O}$ and $\mathrm{Cl}$ (the $\mathrm{C}$ and $\mathrm{Cu}$ signals come from the holey carbon films and the copper

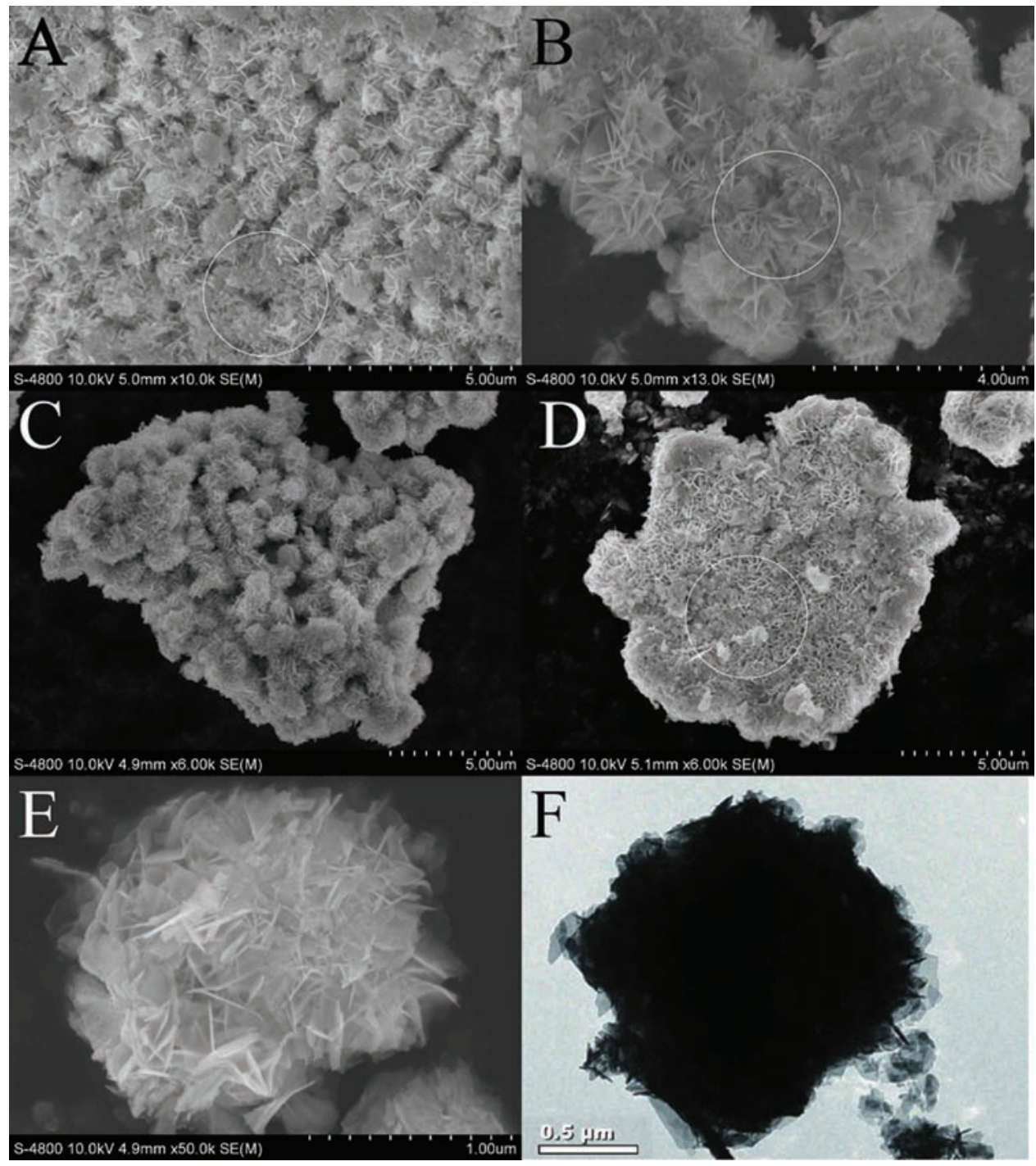

Figure 2. SEM images of (A) BOC0, (B) BOC5, (C) BOC10, (D) BOC 20, enlarged SEM image (E) and TEM image (F) of the BOC10 sample. 
grid, respectively). The atomic ratio of $\mathrm{Bi}: \mathrm{O}: \mathrm{Cl}$ is approximately $1: 1: 1$, further confirming the pure phase of $\mathrm{BiOCl}$ in sample BOC $10 .^{22}$

Based upon the observation so far, we proposed a brief mechanism of $\mathrm{BiOCl}$ microspheres formation. $\mathrm{Bi}\left(\mathrm{NO}_{3}\right)_{3}$. $5 \mathrm{H}_{2} \mathrm{O}$ dissolved into the concentrated $\mathrm{HCl}$ and isolated into $\mathrm{Bi}^{3+}$ ions, which could be stablized under highly acidic environment in the presence of $n$-propanol as cochelating reagents. With the gradual decrease of acidity through the addition of $\mathrm{NaOH}, \mathrm{Bi}^{3+}$ ions inclined to hydrolyze to form $\mathrm{BiOCl}$ crystal nuclei, which continued to grow to $\mathrm{BiOCl}$ nanosheets or nanoflakes, according to their structures features. These $\mathrm{BiOCl}$ nanosheets or nanoflakes were then entirely assembled to provide uniform flow-like microspheres in the presence of sutiable amount of $n$-propanol, though the exact role of $n$-propanol was still unkonwn and required for further investigation. ${ }^{23}$

\subsection{UV-Vis DRS Analysis}

The optical property of as-synthesized $\mathrm{BiOCl}$ samples was measured by UV-vis diffuse reflectance spectra, as shown in Figure 3. The band gap energy of $\mathrm{BiOCl}$ samples could be calculated from the equation $\alpha h v=A\left(h v-E_{g}\right)^{2}$, where $\alpha, v, E_{g}$, and $A$ are absorption coefficient, light frequency, band gap energy, and a constant, respectively. ${ }^{24,25}$ The inset of Figure 3 shows the $(\alpha h v)^{1 / 2}$ versus $(h v)$ curves of the as-synthesized samples and reference $\mathrm{BiOCl}$ sample. By extrapolating the straight portion of $(\alpha h v)^{1 / 2}-$ to- $(h v)$ plot to the $\alpha=0$ point, the band gap of samples BOCO, BOC5, BOC10, and BOC20 are calculated in Table I and are about $3.18 \mathrm{eV}, 3.11 \mathrm{eV}, 3.33 \mathrm{eV}$, and $3.22 \mathrm{eV}$, respectively, which are slightly smaller than the literature reported value $3.4 \mathrm{eV}^{26}$

\section{4. $\mathbf{N}_{2}$ Adsorption-Desorption Isotherms}

Figure 4 showed the nitrogen adsorption-desorption isotherms of as-prepared $\mathrm{BiOCl}$ samples and reference

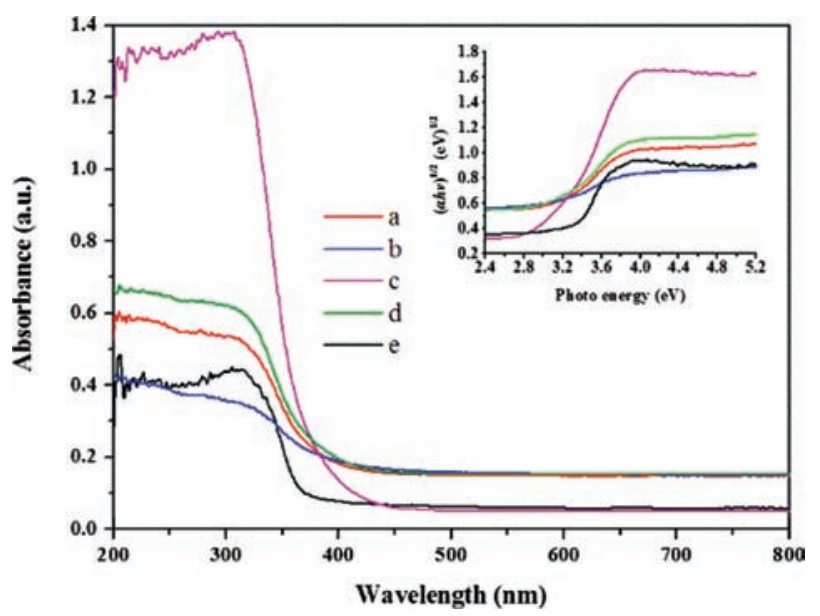

Figure 3. UV-vis diffuse reflectance spectra of as-synthesized samples (a) BOC0; (b) BOC5; (c) BOC10; (d) BOC20; (e) reference BiOCl.
Table I. The BET, $E_{g}$, and $k$ of $\mathrm{BiOCl}$ samples.

\begin{tabular}{lccccc}
\hline Sample & $\begin{array}{c}n \text {-propanol } \\
(\mathrm{mL})\end{array}$ & $\begin{array}{c}\text { Temperature } \\
\left({ }^{\circ} \mathrm{C}\right)\end{array}$ & $\mathrm{BET}\left(\mathrm{m}^{2} \mathrm{~g}^{-1}\right)$ & $E_{g}(\mathrm{eV})$ & $k\left(\mathrm{~min}^{-1}\right)$ \\
\hline BOC0 & 0 & 20 & 27.8659 & 3.18 & 0.023 \\
BOC5 & 5 & 20 & 20.6436 & 3.11 & 0.031 \\
BOC10 & 10 & 20 & 34.3853 & 3.33 & 0.036 \\
BOC20 & 20 & 20 & 31.1271 & 3.22 & 0.034 \\
Reference & $/$ & $/$ & 0.5713 & 3.30 & 0.004 \\
$\quad$ BiOCl & & & & & \\
\hline
\end{tabular}

$\mathrm{BiOCl}$ sample. The isotherms of as-prepared flower-like microspheres can be categorized as type IV observed in the $P / P_{0}$ range of 0.5 to 1.0 and shapes of the hysteresis loop are of type $\mathrm{H} 3$ associated with aggregates of nanosheets, giving rise to slitlike pores, which is consistent well with the SEM results. The specific surface areas of all BiOCl samples were listed in Table I. The sample $\mathrm{BOC} 10$ with uniform $\mathrm{BiOCl}$ flower-like microspheres and slitlike pore nanostructures possessed the largest specific surface area among all $\mathrm{BiOCl}$ samples. As a result, it could afford abundant reactive sites exposing to pollutant molecules. Furthermore, the slitlike pore nanostructures could effectively increase the light harvesting, owing to the light multi-reflection between self-assembled nanosheets. These features might be favorable for the photocatalytic performance. ${ }^{27}$

\subsection{Photocatalytic Activities}

All $\mathrm{BiOCl}$ samples together with $\mathrm{N}$-doped $\mathrm{TiO}_{2}$ were exposed to photocatalytic degradation measurements of $\mathrm{RhB}$ under visible light irradiation. Figure 5(A) showed the temporal evolution of the adsorption spectra of the RhB solution over BOC10 sample. The intensity of the absorption peak of RhB was observed to gradually decrease with prolonging the irradiation time, accompanying a blue shift of the main adsorption peak to lower wavelength, mainly

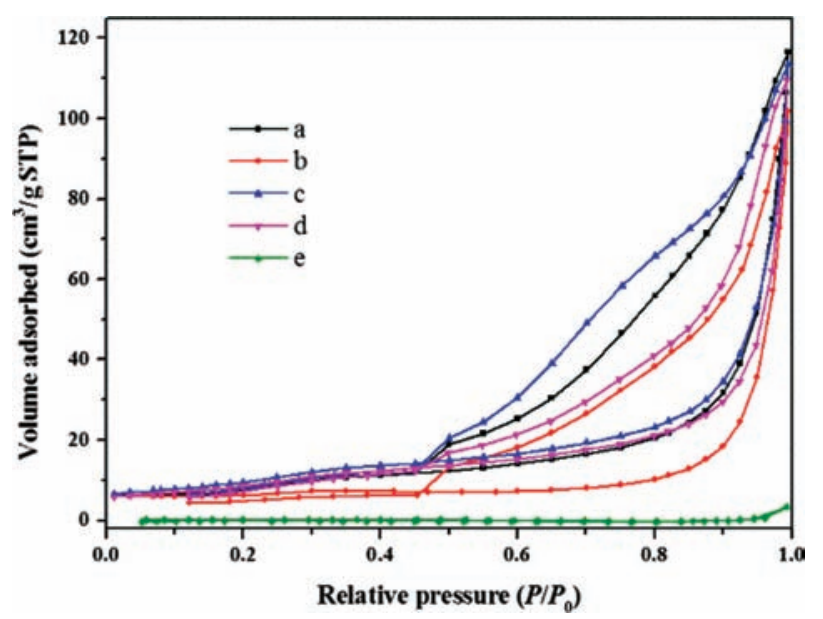

Figure 4. Nitrogen adsorption-desorption isotherms of (a) BOC0; (b) BOC5; (c) BOC10; (d) BOC20; (e) reference BiOCl. 

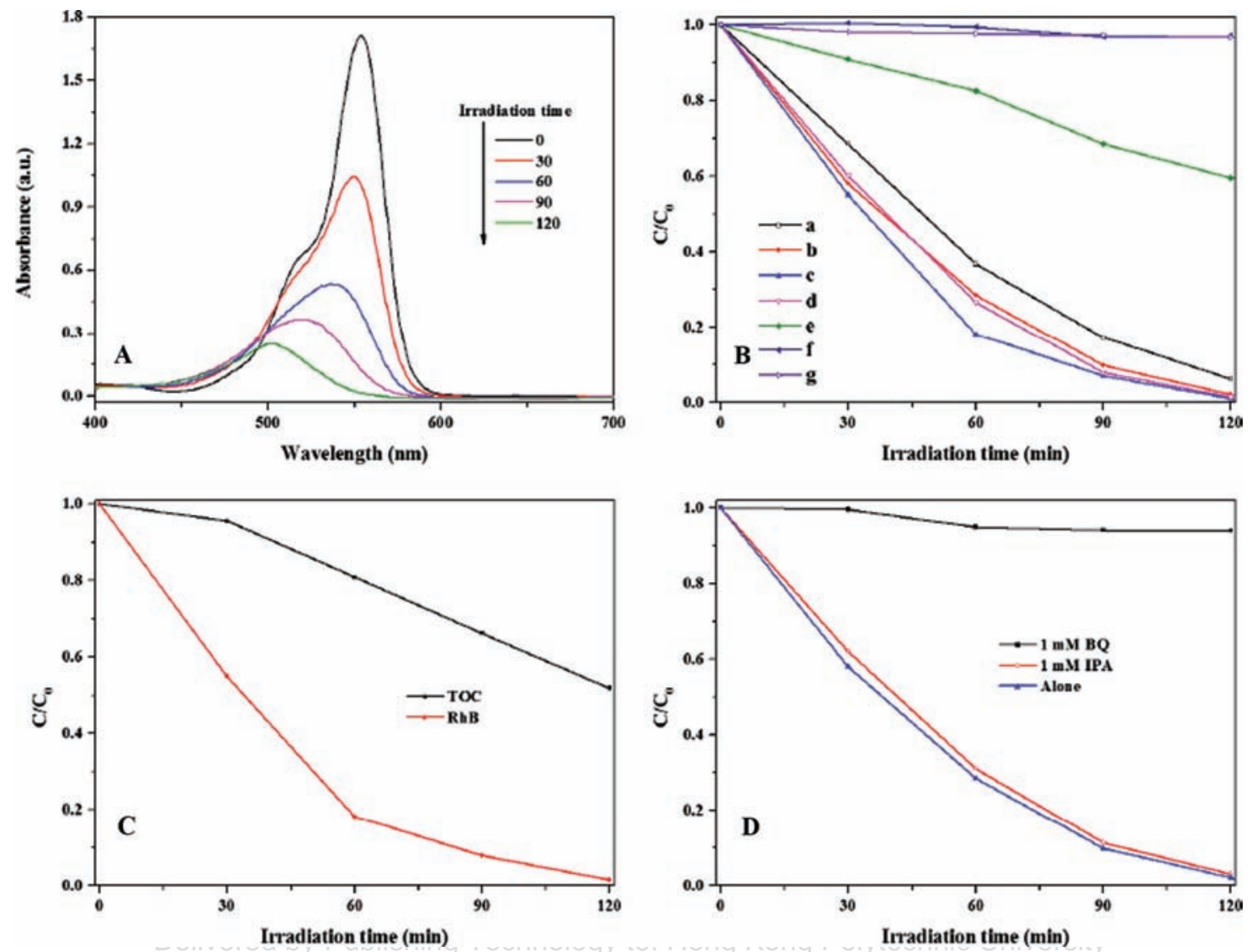

Figure 5. (A) The temporal evolution of the absorbance spectra and photographic image of the $\mathrm{RhB}$ at given time intervals. (B) Photocatalytic activities of $\mathrm{RhB}$ over as-prepared $\mathrm{BiOCl}$ samples: (a) BOC0; (b) BOC5; (c) BOC10; (d) BOC20; (e) reference $\mathrm{BiOCl}$; (f) N-doped $\mathrm{TiO}_{2}$; (g) $\mathrm{Blank}$. (C) RhB and TOC removal rate during the photodegradation over BOC10 sample. (D) Plots of active species capturing for photocatalytic degradation of RhB over BOC10 sample.

attributing to a step-by-step deethylation of $\mathrm{RhB} .{ }^{28}$ During reaction, the color of the suspension changed gradually from initial fuchsia red to light green-yellow after irradiation for $120 \mathrm{~min}$. In order to simplify the analysis, the characteristic absorption peak at $554 \mathrm{~nm}$ was employed to evaluate the photocatalytic degradation.

Direct photolysis of $\mathrm{RhB}$ under the same condition could almost be negligible. ${ }^{29}$ Meanwhile, N-doped $\mathrm{TiO}_{2}$ showed quite low photocatalytic performance even with the increasing irradiation time. Among all $\mathrm{BiOCl}$ samples, the uniform flower-like microspheres BOC10 had the highest photodegration rate, which was about 9 times higher than reference $\mathrm{BiOCl}$ sample, shown in Table I. About $55 \%$ of $\mathrm{RhB}$ could be consumed after 30 min irradiation and the residue could be completely removed after 120 min for sample BOC10. However, only $35 \%$ of $\mathrm{RhB}$ was removed over reference $\mathrm{BiOCl}$ after $120 \mathrm{~min}$, as seen in Figure 5(B). Catalyzed degradation of oxalic acid was also conducted and no concentration decrease was found by TOC analysis, which revealed the degradation by synthesized $\mathrm{BiOCl}$ samples were undergone in a photosensitization manner. In addition, the remarkable variation of the photocatalytic activities between reference $\mathrm{BiOCl}$ and BOC10 sample was mainly attributed to the slitlike pore nanostructures assembled from nanosheets, which could increase both the interfacial surface area between catalysts and pollutant molecules and mass transfer of reactants and products. Meanwhile, the slitlike pore nanostructures between the nanosheets were able to enhance light harvesting, which excited more pollutant molecules to excited states and inject photoinduced electrons to the conduction band of $\mathrm{BiOCl}$, felicitating the photosensitization process.

In Figure 5(C), the variation of TOC was measured over sample BOC10 to determine the complete mineralization of RhB. The TOC decrease only $48 \%$ was gained after $120 \mathrm{~min}$, much lower than the value over $99 \%$ obtained by UV-vis absorption analysis, mainly owning to the complicated mineralization of RhB and only the TOC in the solution counted. ${ }^{30}$

Different active species capturing experiments were carried out and exhibited in Figure 5(D). The degradation efficiency of $\mathrm{RhB}$ kept identical after introduction of IPA, indicating that ${ }^{\circ} \mathrm{OH}$ was not the main reactive radicals. However, the degradation of $\mathrm{RhB}$ over $\mathrm{BiOCl}$ was almost inhibited when $1 \mathrm{mM}$ BQ was added, suggesting that ${ }^{\circ} \mathrm{O}_{2}^{-}$radicals were crucial to the photosensitization process. 


\section{CONCLUSIONS}

In summary, we have facilely constructed uniform $\mathrm{BiOCl}$ flower-like microspheres through simple regulation of $\mathrm{pH}$ value in aqueous with sodium hydroxide in the presence of $n$-propanol. It was obvious that the appropriate amount $n$-propanol was quite important to produce $\mathrm{BiOCl}$ samples containing only flower-like microspheres. Otherwise, some fragments of $\mathrm{BiOCl}$ nanosheets were still coexisted. The uniform $\mathrm{BiOCl}$ flower-like microspheres exhibited enhanced photocatalytic performance against Rhodamine $\mathrm{B}$ in aqueous, mainly attributing to the unique morphologies that ensured the increase of interfacial surface area, mass transfer and light harvesting. The photocatalytic process were undergone through a photosensitization pathway, in which superoxide radicals $\left({ }^{\circ} \mathrm{O}_{2}^{-}\right)$played critical roles.

Acknowledgments: The authors are grateful to the National Natural Science Foundation of China (Grant numbers 21207089 and 41076040), the Innovation Program of Shanghai Municipal Education Commission (Grant number 11YZ113), the project-sponsored by SRF for ROCS, SEM, and 863 project (Grant number 2013AA06A207-1) for financial support.

\section{References and Notes}

1. A. Fujishima and K. Honda, Nature 238, 37 (1972).

2. H. J. Zhang, G. H. Chen, and D. W. Bahnemann, J. Mater. Chem. 19, 5089 (2009).

3. H. T. Li, X. D. He, Z. H. Kang, H. Huang, Y. Liu, J. L. Liu, S. Y. Lian, C. H. A. Tsang, X. B. Yang, and S. T. Lee, Angew. Chem. Int. Ed. 49, 4430 (2010).

4. K. L. Zhang, C. M. Liu, F. Q. Huang, C. Zheng, and W. D. Wang, Appl. Catal. B: Environ. 68, 125 (2006).

5. J. F. Li, Y. C. Zhu, Y. Yan, B. J. Xi, K. B. Tang, and Y. T. Qian, J. Nanosci. Nanotechnol. 12, 2068 (2012).

6. J. X. Xia, J. Zhang, S. Yin, H. M. Li, H. Xu, L. Xu, and Q. Zhang, J. Phys. Chem. Solids 74, 298 (2013).

7. X. P. Lin, T. Huang, F. Q. Huang, W. D. Wang, and J. L. Shi, J. Phys. Chem. B 110, 24629 (2006).

8. Y. J. Liang, C. F. Guo, S. H. Cao, Y. Tian, and Q. Liu, J. Nanosci. Nanotechnol. 13, 919 (2013).
9. J. M. Ma, X. D. Liu, J. B. Lian, X. C. Duan, and W. J. Zheng, Cryst. Growth Des. 10, 2522 (2010).

10. J. Y. Xiong, G. Cheng, R. M. Wang, H. Yang, S. Q. Xiao, and R. Chen, Sci. Adv. Mater. 5, 1024 (2013).

11. Y. Y. Li, J. P. Liu, J. Jiang, and J. G. Yu, Dalton Trans. 40, 6632 (2011).

12. K. Zhang, J. Liang, S. Wang, J. Liu, K. X. Ren, X. Zheng, H. Luo, Y. J. Peng, X. Zou, X. Bo, J. H. Li, and X. B. Yu, Cryst. Growth Des. 12, 793 (2011).

13. Y. Q. Lei, G. H. Wang, S. Y. Song, W. Q. Fan, and H. J. Zhang, CrystEngComm. 11, 1857 (2009).

14. J. M. Song, C. J. Mao, H. L. Niu, Y. H. Shen, and S. Y. Zhang, CrystEngComm. 12, 3875 (2010).

15. Y. Y. Li, J. P. Liu, X. T. Huang, and G. Y. Li, Cryst. Growth Des. 7, 1350 (2007).

16. L. Zhou, W. Z. Wang, H. L. Xu, S. M. Sun, and M. Shang, Chem.Euro. J. 15, 1776 (2009).

17. L. P. Zhu, G. H. Liao, N. C. Bing, L. L. Wang, Y. Yang, and H. Y. Xie, CrystEngComm. 12, 3791 (2010).

18. Y. C. Xie, F. Chang, C. L. Li, J. Chen, J. R. Luo, L. Li, and X. F. Hu, Clean-Soil, Air, Water doi:10.1002/clen.201300014.

19. T. T. Li, L. H. Zhao, Y. M. He, J. Cai, M. F. Luo, and J. J. Lin, Appl. Catal. B: Environ. 129, 255 (2013).

20. P. F. Ji, J. L. Zhang, F. Chen, and M. Anpo, Appl. Catal. B: Environ. 85, 148 (2009).

21. Z. Ling, W. Z. Wang, L. Zhou, M. Shang, and S. M. Sun, Appl. Catal. B: Environ. 90, 458 (2009).

22. L. Chen, S. F. Yin, R. Huang, Y. Zhou, S. L. Luo, and C. T. Au, Catal. Commun. 23, 54 (2012).

23. G. L. Chai, C. S. Lin, J. Wei, M. Y. Zhang, and W. D. Cheng, Phys. Chem. Chem. Phys. 14, 835 (2012)

24. L. S. Zhang, W. Z. Wang, L. Zhou, and H. L. Xu, Small 3, 1618 (2007).

25. S. J. Peng, L. L. Li, P. N. Zhu, Y. Z. Wu, M. Srinivasan, S. G. Mhaisalkar, S. Ramakrishna, and Q. Y. Yan, Chem.-Asian J. 8, 258 (2013).

26. Z. T. Deng, F. Q. Tang, and A. J. Muscat, Nanotechnology 19, 295705 (2008).

27. S. W. Liu, C. Li, J. G. Yu, and Q. J. Xiang, CrystEngComm. 13, 2533 (2011).

28. H. B. Fu, C. S. Pan, W. Q. Yao, and Y. F. Zhu, J. Phys. Chem. B 109, 22432 (2005).

29. J. X. Xia, S. Yin, H. M. Li, H. Xu, L. Xu, and Y. G. Xu, Dalton Trans. 40, 5249 (2011)

30. W. Xiong, Q. D. Zhao, X. Y. Li, and D. K. Zhang, Catal. Commun. 16, 229 (2011).

Received: 9 June 2013. Accepted: 9 October 2013. 